\title{
Optimal Resource Allocation in a Bandwidth Exchange Enabled Relay Network
}

\author{
Muhammad Nazmul Islam, Narayan Mandayam and Sastry Kompella* \\ WINLAB, Rutgers University, Email: mnislam@winlab.rutgers.edu, narayan@winlab.rutgers.edu \\ * Information Technology Division, Naval Research Laboratory, Email: sk@ieee.org
}

\begin{abstract}
We investigate an incentive mechanism called Bandwidth Exchange (BE) for cooperative forwarding where transmission bandwidth is used as a flexible resource. We focus on a network where two nodes, communicating with the base station (BS) / access point (AP), initially get optimal amount of bandwidth based on direct path transmission and then use their individual bandwidths as flexible incentives for two hop relaying. In our proposed scenario, the forwarder node sends its own data along with the data of the sender in exchange for additional transmission bandwidth, provided by the sender. We compare the performance of the proposed mechanism with optimal bandwidth and power allocation based direct transmission. We use sum rate, max-min rate and min-max power as the evaluation criteria and prove the convex/concave nature of the optimization problem formulations. Our numerical analysis shows that the BE based cooperative forwarding extends the coverage in wireless networks when the far node falls in outage under direct transmission. Further, BE significantly improves the max-min rate and minmax power performance of the network.
\end{abstract}

Index Terms-Radio Resource Management, Incentivized Forwarding, Relay Network, Convex Optimization.

\section{INTRODUCTION}

The benefits of cooperative communications [1], [2] have led to a great deal of research in relaying and forwarding [3], [4]. However, few works focus on the cooperation costs that might be significant in a dynamic environment. Existing cooperative forwarding mechanisms include incentivizing nodes using reputation based mechanisms [5], pricing based mechanisms [6] and forwarding games based mechanisms [7]. These mechanisms require a stable economy or a shared understanding of the worth of things that can only be obtained after a long time. Therefore, these pricing mechanisms become ineligible to be implemented in a dynamic tactical wireless network.

In light of this, the authors of [8] have recently developed a Bandwidth Exchange (BE) based cooperative forwarding technique where transmission bandwidth is used as an incentive for relaying. They used a Nash bargaining solution to investigate the rate and coverage gains of $\mathrm{BE}$. We consider a two node network where two nodes initially receive optimal amount of bandwidth based on direct path transmission and connect directly to the base station (BS) / access point (AP). In this context, we focus on a two hop incentivized forwarding scheme where the sender node provides bandwidth to the forwarder node for relaying its data to the AP/BS. We perform optimal bandwidth and power allocation in this BE enabled incentivized forwarding scenario.
There have been some other recent works on bandwidth allocation in two hop forwarding based half duplex relay networks. In [9], the authors focus on sum power minimization in an $\mathrm{N}$ node relay network. The authors of [10] optimize the transmit and relay signals to evaluate the cut-set bounds in a two node multiple input multiple output relay network. In [11], the authors optimize the relay scaling coefficients of the parallel subcarriers in an amplify-and-forward relay network. All these schemes are developed for commercial systems where the relay does not have its own data to communicate with the AP. However, we consider a tactical wireless network where both nodes communicate with the AP. No terminal delays its own transmission to relay messages of other terminals. Thus, the proposed model is suitable to be implemented in a dynamic tactical network.

Numerous utility function optimizations of dynamic spectrum allocation in direct transmission have appeared in the standard literature [12]. The convex/concave nature of these functions have also been proved [13]. In this paper, we compare the performance of our proposed algorithm with that of frequency division multiple access (FDMA) based direct transmission and use max sum rate, max min rate and min max power as the evaluation criteria. The numerical analysis shows that BE extends the coverage of wireless networks when one of the nodes cannot meet its minimum rate requirement through direct transmission. We also show that BE increases the worst case capacity by a factor of up to 2 in max-min rate optimization. Furthermore, we show that BE can save up to 3 $\mathrm{dB}$ power in min-max power optimization formulation.

This paper is organized as follows: In Section II, we present the system model. Section III shows the optimization problem formulations that include max sum rate, max min rate and min max power. Section IV describes the BE based incentivized forwarding protocol. After showing the simulation results in Section V, we conclude the work in Section VI and provide the proofs in the appendices.

\section{SySTEM MODEL}

We consider a two node relay network. We assume half duplex decode and forward relaying in this work. Fig. 1 shows the FDMA based direct transmission and BE based relaying model. Here, Node 0 denotes the AP. Node 1 and 2 denote the two nodes that communicate with AP both in the uplink and downlink. Initially, Node 1 and 2 receive $W_{1}$ and $W_{2}$ bandwidth respectively, obtained through optimal bandwidth 


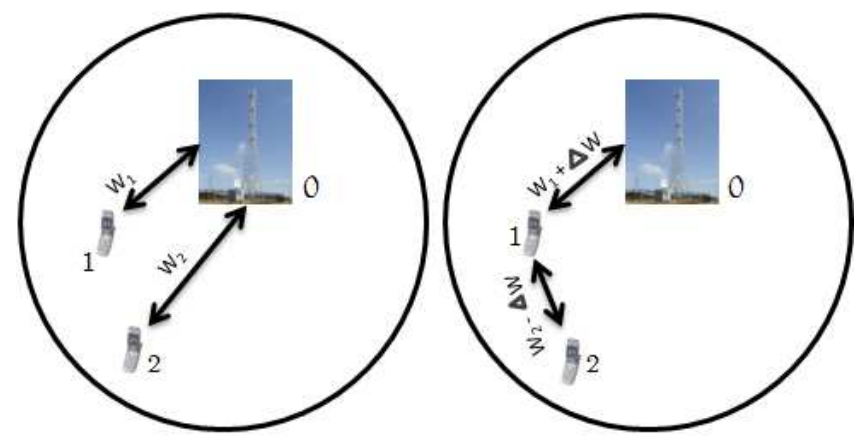

Fig. 1. System Model of FDMA based direct transmission and BE enabled relaying

allocation based on direct path transmission. Let, $\rho_{i j}$ denote the link gain between node $i$ and $j$. Without loss of generality, let us assume, $\rho_{10} \geq \rho_{20}$. In $\mathrm{BE}$ enabled relaying, Node 2 provides a portion of its bandwidth $\delta W$ to Node 1 as an incentive for relaying. Node 1 forwards Node 2's uplink and downlink data along with transmitting its own uplink downlink data. These separate data transmissions take place at different subcarriers and both nodes transmit at the same time slot. We assume $\rho_{i j}=\rho_{j i}$ in our work. The power is denoted by $P$.

We use roman numerals to denote the index of the optimization problem. $P_{i j, I}$ denotes the power used to transmit data between $i$ and $j$ in the $I^{\text {th }}$ optimazion problem. $W_{i j, I I I}$ denotes similar meanings in terms of transmission bandwidth.

The capacity of a path is defined as:

$$
C(W, P, \rho)=W \log _{2}\left(1+\frac{\rho P}{W}\right) \text { bps }
$$

The noise variance is assumed to be equal across all receivers. To illustrate the rate definitions that will be used throughout the paper, let us assume the special case where only uplink transmission is taking place. The rates achieved by the $1^{\text {st }}$ and $2^{\text {nd }}$ nodes through cooperation are given by [8]:

$$
\begin{aligned}
& R_{1}^{\text {coop }, u}=C\left(W_{1}+\delta W, P_{10}, \rho_{10}\right)-R_{2}^{c o o p, u} \\
& R_{2}^{\text {coop }, u}=\min \left(C\left(W_{2}-\delta W, \rho_{21}, P_{21}\right)\right. \text {, } \\
& \left.C\left(W_{1}+\delta W, P_{10}, \rho_{10}\right)-R_{1}^{c o o p}, u\right)
\end{aligned}
$$

We use $u$ and $d$ in the superscript to represent uplink and downlink transmission respectively. Based on (2) and (3), we can see that $R_{1}^{c o o p, u}$ and $R_{2}^{c o o p, u}$ can take on numerous values.

Let us consider a scenario where $R_{2}^{\text {coop }, u}<$ $C\left(W_{2}-\delta W, \rho_{21}, P_{21}\right)$. It can be clearly seen that Path 21 has been allocated more bandwidth than it needs to maintain the data rate of node $2, R_{2}^{c o o p}, u$. Hence, the system can transfer some bandwidth from Path 21 to Path 10 and thus increase the data rate of Node 1 without affecting that of Node 2. Therefore, we assume that Path 10 always carries at least $C_{2}\left(W_{2}-\delta W, \rho_{21}, P_{21}\right)$ data dedicated for Node 2 . This assumption leads to the following form of $R_{1}^{c o o p}$ and $R_{2}^{\text {coop }}$ :

$$
R_{1}^{\text {coop }, u}=C\left(W_{1}+\delta W, P_{10}, \rho_{10}\right)-C\left(W_{2}-\delta W, \rho_{21}, P_{21}\right)
$$

$$
R_{2}^{\text {coop }, u}=C\left(W_{2}-\delta W, \rho_{21}, P_{21}\right)
$$

\section{Optimization PROBLEM Formulations}

\section{A. Sum rate maximization}

The sum-rate maximization problem in a two node BE based relay network is formulated as,

$$
\begin{gathered}
\max . R_{1}^{\text {coop }, u}+R_{2}^{\text {coop }, u}+R_{1}^{\text {coop }, d}+R_{2}^{\text {coop }, d} \\
R_{1}^{\text {coop }, u} \geq R_{1, \text { min }}^{u}, R_{1}^{\text {coop }, d} \geq R_{1, \text { min }}^{d} \\
R_{2}^{\text {coop }, u} \geq R_{2, \text { min }}^{u}, R_{2}^{\text {coop }, d} \geq R_{2, \text { min }}^{d}
\end{gathered}
$$

$R_{i, \text { min }}^{u}$ and $R_{i, \min }^{d}$ denote the minimum uplink and downlink required rates at node $i$.

Using (4) and (5), the sum rate maximization problem subject to the total available bandwidth, node's maximum power and individual rate constraints takes the following form:

\section{Problem I}

$\max . W_{10, I} \log \left(1+\frac{\rho_{10} P_{10, I}}{W_{10, I}}\right)+W_{01, I} \log \left(1+\frac{\rho_{01} P_{01, I}}{W_{01, I}}\right)$

$$
\begin{gathered}
\text { s.t. } W_{21, I} \log \left(1+\frac{\rho_{21} P_{21, I}}{W_{21, I}}\right) \geq R_{2, \text { min }}^{u} \\
W_{12, I} \log \left(1+\frac{\rho_{12} P_{12, I}}{W_{12, I}}\right) \geq R_{2, \text { min }}^{d}
\end{gathered}
$$

$W_{10, I} \log \left(1+\frac{\rho_{10} P_{10, I}}{W_{10, I}}\right) \geq R_{1, \text { min }}^{u}+W_{21, I} \log \left(1+\frac{\rho_{21} P_{21, I}}{W_{21, I}}\right)$

$W_{01, I} \log \left(1+\frac{\rho_{01} P_{01, I}}{W_{01, I}}\right) \geq R_{1, \text { min }}^{d}+W_{12, I} \log \left(1+\frac{\rho_{12} P_{12, I}}{W_{12, I}}\right)$

$$
W_{10, I}+W_{12, I}+W_{01, I}+W_{21, I} \leq W_{1}^{o p t}+W_{2}^{o p t}
$$

$P_{12, I}+P_{10, I} \leq P_{1}^{\max }, P_{01, I} \leq P_{0}^{\max }, P_{21, I} \leq P_{2}^{\max }$

Var. $P_{12, I}, P_{21, I}, P_{01, I}, P_{10, I}, W_{12, I}, W_{21, I}, W_{01, I}, W_{10, I}$

Here, $P_{i}^{\max }$ is the maximum transmission power at node/AP $i$. $W_{1}^{o p t}$ and $W_{2}^{o p t}$ denote the optimal bandwidth allocated to Node 1 and 2 respectively. Here $W_{1}^{o p t}$ and $W_{2}^{o p t}$ are obtained based on optimal bandwidth allocation to maximize sum rate in direct path transmission. Equation (7f) mentions that the total bandwidth used in the two hop relaying scenario cannot exceed the summation of the initial bandwidths allocated to Node 1 and Node 2. Equation (7d) says that the capacity of Path 10 should be greater than the summation of the $1^{\text {st }}$ node's minimum uplink data rate and the capacity of Path 21 . Equation (7e) holds similar meaning in the downlink direction.

Theorem 1: The optimal solution of Problem I occurs at the following point,

$$
\begin{aligned}
& W_{21, I}^{*} \log \left(1+\frac{\rho_{21} P_{21, I}^{*}}{W_{21, I}^{*}}\right)=R_{2, \text { min }}^{u} \\
& W_{12, I}^{*} \log \left(1+\frac{\rho_{12} P_{12, I}^{*}}{W_{12, I}^{*}}\right)=R_{2, \text { min }}^{d}
\end{aligned}
$$

Proof: See Appendix A. 


\section{B. Minimum rate maximization}

The general form of minimum rate maximization problem in a BE enabled relay network can be written as,

$$
\max . \min .\left(R_{1}^{c o o p, u}, R_{2}^{c o o p, u}, R_{1}^{c o o p, d}, R_{2}^{c o o p, d}\right)
$$

1) Optimality Proof:: Using (4), (5) and (9), the minimum rate maximization problem can be formulated as follows,

\section{Problem II-A}

$\operatorname{max.} \min .\left(R_{10, I I}-R_{21, I I}, R_{21, I I}, R_{01, I I}-R_{12, I I}, R_{12, I I}\right)$

$$
\text { s.t. } W_{10, I I}+W_{01, I I}+W_{12, I I}+W_{21, I I}=W_{1}^{o p t}+W_{2}^{o p t}
$$

$$
P_{12, I I}+P_{10, I I} \leq P_{1}^{\max }, P_{01, I I} \leq P_{0}^{\max }, P_{21, I I} \leq P_{2}^{\max }
$$

$\operatorname{Var.} P_{12, I I}, P_{21, I I}, P_{01, I I}, P_{10, I I}, W_{12, I I}$,

$$
W_{21, I I}, W_{01, I I}, W_{10, I I}
$$

Here,

$$
\begin{aligned}
& R_{10, I I}=W_{10, I I} \log \left(1+\frac{\rho_{10} P_{10, I I}}{W_{10, I I}}\right) \\
& R_{21, I I}=W_{21, I I} \log \left(1+\frac{\rho_{21} P_{21, I I}}{W_{21, I I}}\right) \\
& R_{01, I I}=W_{01, I I} \log \left(1+\frac{\rho_{01} P_{01, I I}}{W_{01, I I}}\right) \\
& R_{12, I I}=W_{12, I I} \log \left(1+\frac{\rho_{12} P_{12, I I}}{W_{12, I I}}\right)
\end{aligned}
$$

Here $W_{1}^{o p t}$ and $W_{2}^{o p t}$ are obtained based on optimal bandwidth allocation to maximize the minimum rate in direct path transmission. Problem II-A is nonconcave since the optimization function contains the difference of concave functions. However, due to the power and bandwidth constraints and the nature of the max-min formulation, the following equality holds at the optimal solution of Problem II-A:

$$
\begin{gathered}
R_{10, I I}-R_{21, I I}=R_{21, I I}=R_{01, I I}-R_{12, I I}=R_{12, I I} \\
R_{10, I I}=2 * R_{21, I I}=R_{01, I I}=2 * R_{12, I I}
\end{gathered}
$$

The same set of $P$ and $W$ variables will maintain both (12) and (13). This leads us to investigate a new optimization problem that has the same constaints as Problem II. Equation (13) motivates us to replace the non-concave optimization function of Problem II-A with the following one:

\section{Problem II-B}

$$
\begin{aligned}
& \max . \min .\left(R_{10, I I}, 2 * R_{21, I I}, R_{01, I I}, 2 * R_{12, I I}\right) \\
& \text { s.t. } W_{10, I I}+W_{01, I I}+W_{12, I I}+W_{21, I I}=W_{1}^{o p t}+W_{2}^{o p t} \\
& P_{12, I I}+P_{10, I I} \leq P_{1}^{\max }, P_{01, I I} \leq P_{0}^{\max }, P_{21, I I} \leq P_{2}^{\max }
\end{aligned}
$$

$$
\begin{gathered}
\operatorname{Var} . P_{12, I I}, P_{21, I I}, P_{01, I I}, P_{10, I I}, W_{12, I I}, \\
W_{21, I I}, W_{01, I I}, W_{10, I I}
\end{gathered}
$$

The optimal solutions of Problem II-A and II-B are different. However, (12) and (13) suggest that the optimal variables of Problem IIA and IIB are same. Now, (14a) is the minimum of a set of concave functions and therefore concave. Since the constraints (14b) and (14c) are linear, Problem II-B is a concave maximization problem. Thus, we can attain the optimal solution of Problem II-A using the global optimal variables of Problem II-B.

\section{Maximum power minimization}

The min-max power optimization problem of a BE enabled relay network can be formulated as,

\section{Problem III}

$$
\begin{gathered}
\text { min. max. }\left(P_{10, I I I}, P_{21, I I I}, P_{01, I I I}, P_{12, I I I}\right) \\
\text { s.t. } W_{10, I I I} \log \left(1+\frac{\rho_{10} P_{10, I I I}}{W_{10, I I I}}\right)-R_{2, \text { min }}^{u} \geq R_{1, \text { min }}^{u} \\
W_{21, I I I} \log \left(1+\frac{\rho_{21} P_{21, I I I}}{W_{21, I I I}}\right) \geq R_{2, \text { min }}^{u} \\
W_{01, I I I} \log \left(1+\frac{\rho_{01} P_{01, I I I}}{W_{01, I I I}}\right)-R_{2, \text { min }}^{d} \geq R_{1, \text { min }}^{d} \\
W_{12, I I I} \log \left(1+\frac{\rho_{12} P_{12, I I I}}{W_{12, I I I}}\right) \geq R_{2, m i n}^{d} \\
W_{10, I I I}+W_{12, I I I}+W_{01, I I I}+W_{21, I I I} \leq W_{1}^{\text {opt }}+W_{2}^{o p t} \\
\quad \operatorname{Var.~} P_{12, I I}, P_{21, I I}, P_{01, I I}, P_{10, I I}, W_{12, I I} \\
W_{21, I I}, W_{01, I I}, W_{10, I I}
\end{gathered}
$$

Here $W_{1}^{o p t}$ and $W_{2}^{o p t}$ are obtained based on optimal bandwidth allocation to minimize the maximum power in direct path transmission.

The optimization function of Problem III is a minimization of linear, i.e., convex, functions. The inequality constraints are either convex or linear. Therefore, Problem III is a convex minimization problem.

\section{BE BASED INCENTIVIZED FORWARDING PROTOCOL}

- The BS sends channel training symbols to both Node 1 and 2 .

- Nodes estimate their link gains, $\rho_{10}$ and $\rho_{20}$, and feed back these information to the BS. We assume perfect channel estimation and error-free feedback.

- The BS allocates $W_{1}^{o p t}$ and $W_{2}^{o p t}$ to Node 1 and Node 2 respectively. $W_{1}^{o p t}$ and $W_{2}^{o p t}$ are obtained through optimal bandwidth allocation based on $\rho_{10}$ and $\rho_{20}$.

- Node 1 and 2 send an omnidirectional signal containing their direct link gains, X-Y coordinates and allocated bandwidths to each other.

- In a path-loss based channel model, Node 1 and 2 estimate $\rho_{12}$, inter-node link gain, using the distances between the nodes. In a large and small scale fading model, $\rho_{12}$ is estimated using past history and a low pass filter. 


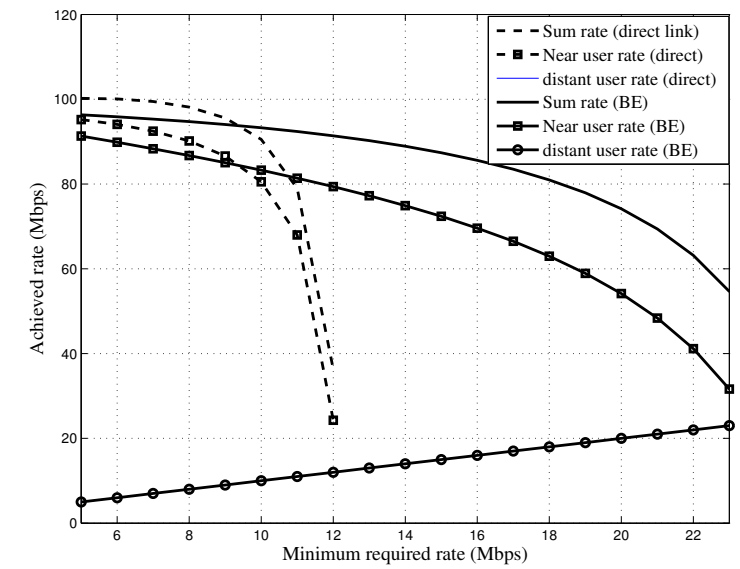

Fig. 2. Sumrate in FDMA and BE $\left(P_{10}=P_{21}=20 \mathrm{dBm}, d_{10}=150 \mathrm{~m}\right.$, $\left.d_{12}=300 \mathrm{~m}, d_{20}=400 \mathrm{~m}, R_{1, \min }=R_{2, \min }\right)$

- Using $\rho_{10}, \rho_{20}$ and $\rho_{12}$, both Node 1 and 2 can solve Problem I-III on their own. The sender node informs the forwarder node about the frequency range of the additional bandwidth.

\section{Numerical Results}

In our simulation model, we assume the link gains to take the form, $\rho_{i j}=k d_{i j}^{-3}$, where the path loss exponent is chosen as 3 and $d_{i j}$ is the distance between the $i^{\text {th }}$ transmitter and $j^{\text {th }}$ receiver. $k$ is the proportionality constant that also captures the noise spectral density and is set to $k=6 \times 10^{6} \mathrm{MHz} *$ $\mathrm{m}^{3} / \mathrm{mW}$ [8]. We assume optimal initial resource allocation, i.e., nodes start with optimal bandwidth, allocated based on direct path transmission.

\section{A. Sumrate maximization}

Fig. 2 shows the comparison of direct transmission and BE based relaying. We consider fixed transmission power and uplink transmission only in this case. We assume, $P_{10}=$ $P_{21}=20 \mathrm{dBm}, d_{10}=150 \mathrm{~m}, d_{12}=300 \mathrm{~m}, d_{20}=400 \mathrm{~m}$, $R_{1, \min }=R_{2, \min }$. The sum rate and individual user rates are obtained for different minimum rates. The total bandwidth is $40 \mathrm{MHz}$. When minimum rate ranges from 1 to $12 \mathrm{Mbps}$, Node 1 and 2 receive optimal bandwidth to maximize sum rate through direct transmission. In BE based relaying, in this range, nodes start with the initial bandwidth allocation and then transfer bandwidth among themselves to maximize sum rate. The optimal bandwidth allocation in direct transmission becomes infeasible when $R_{\text {min }}$ exceeds $12 \mathrm{Mbps}$. Therefore, when $R_{\text {min }}$ ranges from $13 \mathrm{Mbps}$ to $25 \mathrm{Mbps}$, we assume $W_{1}^{o p t}+W_{2}^{o p t}=40 M H z$, i.e., the total available bandwidth, in (7f) to solve the sum rate maximization problem in a $\mathrm{BE}$ enabled network.

From $R_{\min }=13 \mathrm{Mbps}$, the distant node falls in outage under direct transmission. However, BE based relaying provides feasible transmission up to $R_{\text {min }}=23 M b p s$. Thus BE based relaying outperforms FDMA based direct transmission

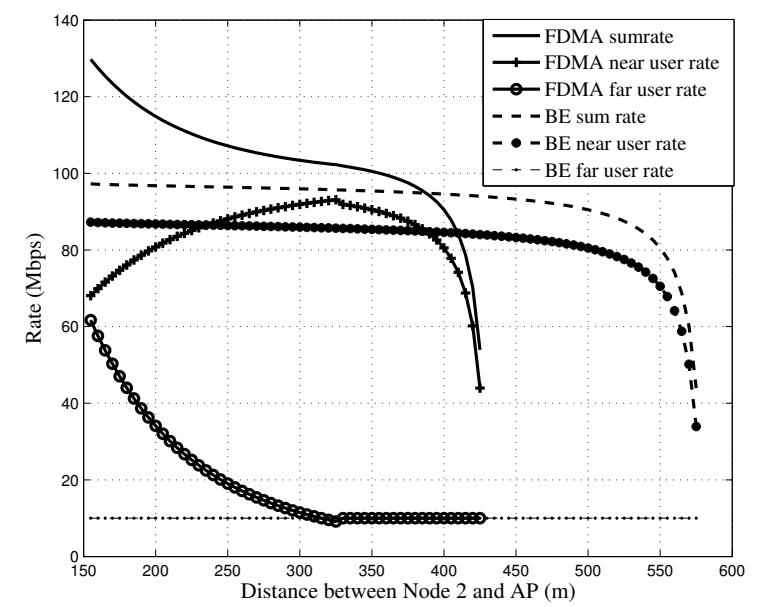

Fig. 3. Coverage Extension in BE enabled relay networks $\left(P_{10}=P_{21}=\right.$ $\left.20 d B m, d_{12}=\alpha, d_{20}=d_{10}+\alpha, R_{1, \min }=R_{2, \min }\right)$

when the distant node falls in outage. Fig. 2 also shows that the distant node exactly gets the minimum required rate at feasible solutions.

Fig. 3 compares the coverage of BE enabled relay networks with direct transmission. In Fig. 3, we used $d_{12}=\alpha$, $d_{20}=d_{10}+\alpha$, i.e., Node 1 is placed in the straight line connecting Node 2 and AP. Fig. 3 shows that FDMA based direct transmission becomes infeasible as the distance between Node 2 and AP exceeds $425 \mathrm{~m}$. On the other hand, BE provides coverage up to $575 \mathrm{~m}$. Fig. 3 also shows that FDMA performs better than $\mathrm{BE}$ when Node 2 is closer to the BS. This suggests that $\mathrm{BE}$ is most advantageous when the distant node falls in outage though direct transmission.

\section{B. Minimum rate maximization}

Fig. 4 shows the maxmin rate comparison in the uplink data transmission of FDMA based direct transmission and $\mathrm{BE}$ based relaying. Here, $P_{10}=P_{21}=20 \mathrm{dBm}, d_{10}=150 \mathrm{~m}$, $d_{12}=300 \mathrm{~m}, d_{20}=400 \mathrm{~m}$. For each total bandwidth, at first, bandwidth is optimally allocated to maximize the minimum rate for direct path transmission. Thereafter, nodes exchange bandwidth among themselves using the protocol of Sec. IV to solve Problem II, i.e., nodes maximize the minimum rate in the BE enabled network. Fig. 4 shows that the BE based relaying improves the maxmin rate by a factor of 2 in this scenario.

\section{Maximum power minimization}

Fig. 5 compares the minmax power of a BE based relay network with that of a FDMA based direct transmission. We assume, $R_{1, \min }=R_{2, \min }=R_{\text {min }}, d_{10}=150 \mathrm{~m}$, $d_{12}=300 \mathrm{~m}, d_{20}=400 \mathrm{~m}$. Here, the total bandwidth, $B=40 M H z$. For each minimum required rate, bandwidth is optimally allocated to minimize the maximum transmission power for direct path transmission. Thereafter, nodes exchange bandwidth among themselves using the protocol of Section IV 


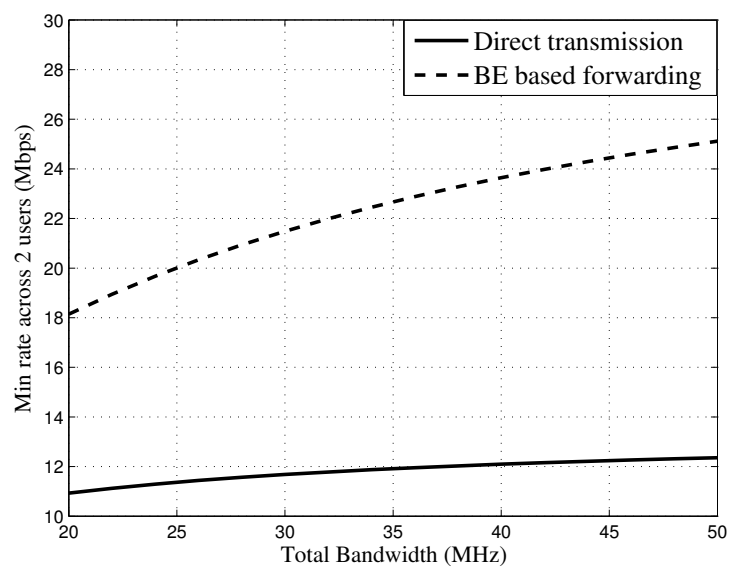

Fig. 4. Minrate maximization in BE and FDMA $\left(P_{\max }=20 \mathrm{dBm}, d_{10}=\right.$ $\left.150 m, d_{20}=400 m, d_{12}=300 m\right)$

to solve Problem III, i.e., minimize the maximum power in the BE enable network. Fig. 5 shows that BE can lead to almost 3 $\mathrm{dB}$ power reduction. Thus, $\mathrm{BE}$ enabled relaying can increase the battery life in a tactical network.

\section{CONCLUSION}

In this paper we considered a BE enabled relay network and studied the resource allocation problems of maximum sum rate, max min rate and min max power. We proved the concavity/convexity of these problems. Our numerical results showed that BE is particularly advantageous when the far node cannot meet its minimum data rate requirement through direct transmission. We also show that BE can significantly improve the maxmin rate and minmax power performance of the network.

We plan to extend the proposed framework to an $\mathrm{N}$ node network. Any node can act as a relay in an $\mathrm{N}$ node network if its inclusion in the relay set increases the system utility. Therefore, the selection of the optimal set of relay nodes will be the area of our future research. Transmission time can also be used as a flexible resource for relaying in tactical wireless networks. We plan to investigate time exchange enabled relaying as an extension of this work, as well.

Our simulations results only involve path loss based channel models. However, our work can also be extended to large and small scale fading scenarios. Numerical simulations involving more realistic channel models remain another area of future work.

\section{ACKNOWLEDGEMENTS}

This work is supported by the Office of Naval Research under grant N00014-11-1-0132.

\section{APPENDIX A}

PROOF OF THEOREM 1

Lemma 1 : Problem $I$ is non-concave due to the constraints (7d) and (7e).

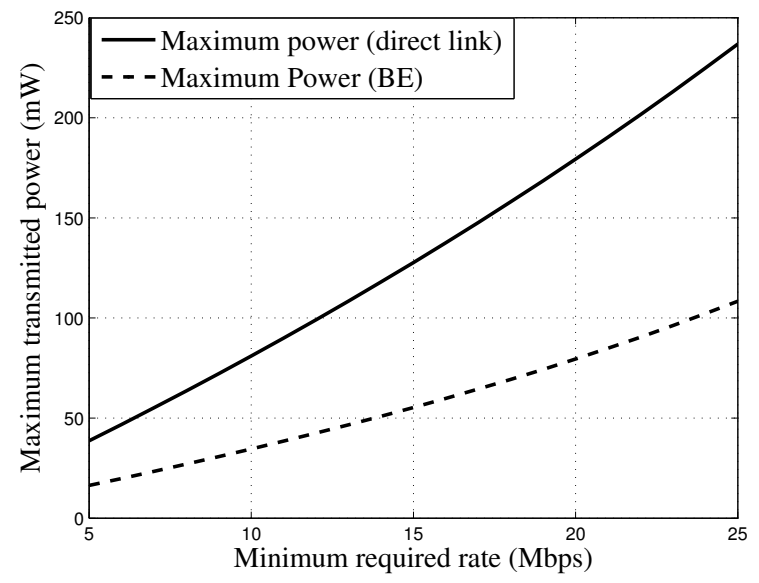

Fig. 5. Maximum power minimization in BE and FDMA $\left(d_{10}=150 \mathrm{~m}\right.$, $\left.d_{20}=400 m, d_{12}=300 m, B=40 M H z\right)$

Proof: We know that $\log ($.$) is a concave function. Since$ $1+\rho P$ is linear, $\log (1+\rho P)$ is concave in $P . W \log \left(1+\frac{\rho P}{W}\right)$ is a perspective function of $W$ and therefore concave in $P, W$ [14]. We also know that the sum of concave functions are concave. Hence, (7a) is a concave function and (7b) and (7c) represent convex constraints. The constraints $(7 \mathrm{f})$ and $(7 \mathrm{~g})$ are linear. The constraints $(7 \mathrm{~d})$ and $(7 \mathrm{e})$ contain difference of concave functions and therefore non-convex [14]. Hence, Problem I becomes a non-concave maximization problem due to the constraints (7d) and (7e).

To find the solution of Problem I, we consider a different optimization problem, where we focus on sum rate maximization in the uplink, and nodes transmit at their individual maximum transmission power.

\section{Problem IV}

$$
\max . W_{10, I V} \log \left(1+\frac{\rho_{10} P_{1}^{\max }}{W_{10, I V}}\right)
$$

$$
\begin{gathered}
\text { s.t. } W_{10, I V} \log \left(1+\frac{\rho_{10} P_{1}^{\max }}{W_{10, I V}}\right) \geq R_{1, \min }^{u}+R_{2, \min }^{u} \\
W_{21, I V} \log \left(1+\frac{\rho_{21} P_{2}^{\max }}{W_{21, I V}}\right) \geq R_{2, \min }^{u} \\
W_{10, I V}+W_{21, I V} \leq W_{1}^{o p t}+W_{2}^{o p t} \\
\text { Var. } W_{10, I V}, W_{21, I V}
\end{gathered}
$$

In Problem IV, we notice that the optimization function is concave and the constraints are either convex or linear. Therefore, Problem IV is a concave maximization problem.

Lemma 2 : The optimal solution of Problem IV occurs at the following point,

$$
\begin{gathered}
W_{21, I V}^{*} \log \left(1+\frac{\rho_{21} P_{2}^{\max }}{W_{21, I V}^{*}}\right)=R_{2, \text { min }}^{u} \\
W_{10, I V}^{*}=W_{1}^{\text {opt }}+W_{2}^{\text {opt }}-W_{21, I V}^{*}
\end{gathered}
$$


Proof: We will prove lemma 2 by contradiction. Let $W_{21, I V}$ be any solution of Problem IV. If $W_{21, I V}<W_{21, I V}^{*}$,

$$
\begin{aligned}
W_{21, I V} \log \left(1+\frac{\rho_{21} P_{2}^{\max }}{W_{21, I V}}\right) & <W_{21, I V}^{*} \log \left(1+\frac{\rho_{21} P_{2}^{\max }}{W_{21, I V}^{*}}\right) \\
& =R_{2, \text { min }}^{u}
\end{aligned}
$$

which violates constraint (16c). On the other hand, if $W_{21, I V}>W_{21, I V}^{*}$, then $W_{10, I V}<W_{10, I V}^{*}$. This means that,

$W_{10, I V} \log \left(1+\frac{\rho_{10} P_{1}^{\max }}{W_{10, I V}}\right)<W_{10, I V}^{*} \log \left(1+\frac{\rho_{10} P_{1}^{\max }}{W_{10, I V}^{*}}\right)$

Therefore, the optimal bandwidth allocation of Problem IV is defined by the analytical solution of Lemma 2 .

Lemma 2 suggests that Node 2 only gets its minimum required rate at the optimal point. This motivates us to consider a separate optimization problem that has the same objective function and the convex constraints of Problem I ((7b), (7c), (7f), (7g)). However, the non-convex constraints of Problem I get replaced with that of the convex ones.

\section{Problem V}

$\max . W_{10, V} \log \left(1+\frac{\rho_{10} P_{10, V}}{W_{10, V}}\right)+W_{01, V} \log \left(1+\frac{\rho_{01} P_{01, V}}{W_{01, V}}\right)$

$$
\begin{gathered}
\text { s.t. } W_{21, V} \log \left(1+\frac{\rho_{21} P_{21, V}}{W_{21, V}}\right) \geq R_{2, \text { min }}^{u} \\
W_{12, V} \log \left(1+\frac{\rho_{12} P_{12, V}}{W_{12, V}}\right) \geq R_{2, \text { min }}^{d} \\
W_{10, V} \log \left(1+\frac{\rho_{10} P_{10, V}}{W_{10, V}}\right) \geq R_{1, \text { min }}^{u}+R_{2, \text { min }}^{u} \\
W_{01, V} \log \left(1+\frac{\rho_{01} P_{01, V}}{W_{01, V}}\right) \geq R_{1, \text { min }}^{d}+R_{2, \text { min }}^{d} \\
W_{10, V}+W_{12, V}+W_{01, V}+W_{21, V} \leq W_{1}^{\text {opt }}+W_{2}^{\text {opt }} \\
P_{12, V}+P_{10, V} \leq P_{1}^{\text {max }}, P_{01, V} \leq P_{0}^{\text {max }}, P_{21, V} \leq P_{2}^{\text {max }}
\end{gathered}
$$

$$
\begin{gathered}
\text { Var. } P_{12, V}, P_{21, V}, P_{01, V}, P_{10, V}, W_{12, V} \\
W_{21, V}, W_{01, V}, W_{10, V}
\end{gathered}
$$

Note that, apart from the the optimization problem index, (21a), (21b), (21c), (21f) and (21g) are same as (7a), (7b), (7c), (7f) and (7g). However, in (21d) and (21e), we have assumed that the capacity of path 10(01) should be greater than the summation of the uplink(downlink) data rates of the two nodes. Both of these constraints are convex. Therefore, the converted optimization problem is a concave maximization one.

Using the reasoning of Lemma 2, it can be easily shown that Node 2 will only get its minimum required rate in the uplink and downlink at the optimal solution of Problem V. Thus,

$$
W_{21, V}^{*} \log \left(1+\frac{\rho_{21} P_{21, V}^{*}}{W_{21, V}^{*}}\right)=R_{2, \min }^{u}
$$

$$
W_{12, V}^{*} \log \left(1+\frac{\rho_{12} P_{12, V}^{*}}{W_{12, V}^{*}}\right)=R_{2, \text { min }}^{d}
$$

Let $p_{I}^{*}$ and $p_{V}^{*}$ be the optimal solutions of Problem I and Problem V respectively.

Lemma 3: $p_{I}^{*}=p_{V}^{*}$.

Proof: Every feasible solution of Problem I maintains (21d) and (21e). Therefore, the feasible set of Problem I is a subset of that of Problem V. Since both Problem I and Problem V are maximization problems, $p_{I}^{*} \leq p_{V}^{*}$.

Equation (22a) and (22b) show that the rate constraints of Node 2 are met tightly at the optimal solution. Therefore, the optimal solution of Problem V, $p_{V}^{*}$, is a feasible solution to Problem I. Hence, by definition, $p_{V}^{*} \leq p_{I}^{*}$. So, $p_{I}^{*}=p_{V}^{*}$.

Therefore, the optimal solution of Problem I maintains (22a) and $(22 b)$.

This proves Theorem 1 .

\section{REFERENCES}

[1] A. Sendonaris, E. Erkip, and B. Aazhang, "User cooperation diversity - part i: System description," IEEE Trans. Commun., vol. 51(11), pp. 1927-1938, Nov. 2003.

[2] J. N. Laneman, D. N. C. Tse, and G. Wornell, "Cooperative diversity in wireless networks : efficient protocols and outage behavior," IEEE Trans. Info. Theory, vol. 50(12), pp. 3062-3080, Dec. 2004.

[3] A. Host-Madsen and J. Zhang, "Capacity bounds and power allocation for the wireless relay channel," IEEE Trans. Info. Theory, vol. 51(6), pp. 2020-2040, June 2005.

[4] L. Lai, K. Liu, and H. El Gamal, "The three node wireless network: Achievable rates and cooperation strategies," IEEE Trans. Info. Theory, vol. 52(3), pp. 805-828, Mar. 2006.

[5] S. Buchegger and J.-Y Le Boudec, "Self-policing mobile ad hoc networks by reputation systems," IEEE Communication Magazine, vol. 43, pp. 101-107, July 2007.

[6] O. Ileri, S.-C. Mau, and N. Mandayam, "Pricing for enabling forwarding in self-configuring ad hoc networks," IEEE JSAC, vol. 23, pp. 151-162, Jan. 2005.

[7] M. Felegyhazi, J. P. Hubaux, and L. Buttyan, "Nash equilibria of packet forwarding strategies in wireless ad hoc networks," IEEE Trans. on Mobile Computing, vol. 5, pp. 463-475, May 2006.

[8] D. Zhang, R. Shinkuma, and N. B. Mandayam, "Bandwidth exchange: An energy conserving incentive mechanism for cooperation," IEEE Trans. Wireless Comm, vol. 9(6), pp. 2055-2065, June 2010.

[9] N. Krishnan, J. S. Panchal, N. B. Mandayam, and R. D. Yates, "Bandwidth sharing in lte-a relaying systems," in Proc. IEEE Allerton'2010, Sept. 2010, pp. 1125-1128.

[10] C. T. K. Ng and G. J. Foschini, "Transmit signal and bandwidth optimization in multiple-antenna relay channels," submitted for publication, http://arxiv.org/abs/1001.2938 [cs.IT].

[11] W. Zhang, U. Mitra, and M. Chiang, "Optimization of amplify-andforward multicarrier two-hop transmission," submitted for publication, http://www.princeton.edu/ chiangm/afmulticarrier.pdf.

[12] G. Song and Y. Li, "Cross-layer optimization for ofdm wireless networks - part ii : Algorithm development," IEEE Trans. Wireless Comm, vol. 4(2), pp. 614-624, Mar. 2005.

[13] Z. Luo and S. Zhang, "Dynamic spectrum management: Complexity and duality,' IEEE Trans. Wireless Comm, vol. 2(1), pp. 57-73, Feb. 2008.

[14] S. Boyd and L. Vandenberghe, Convex Optimization, Cambridge University, Cambridge, UK, 2004. 